\title{
Estrés, Sobrecarga Y Ansiedad En Cuidadoras Primarias De Niños Que Padecen Leucemia En Hidalgo
}

\author{
Karla Salinas Ríos, Licenciada \\ Rodolfo de Jesús Acevedo Martínez, Licenciado \\ Arturo Del Castillo Arreola, Doctor \\ Rebeca María Elena Guzmán Saldaña, Doctora \\ José Esael Pineda Sánchez, Doctor \\ Universidad Autónoma del Estado de Hidalgo, México
}

doi: 10.19044/esj.2017.v13n24p79 URL:http://dx.doi.org/10.19044/esj.2017.v13n24p79

\begin{abstract}
Leukemia is the type of cancer that occurs more in children and teenagers. The chronic-degenerative nature of the disease makes that the minors need someone responsible for their care known as informal primary caregiver. Generally, the primary caregiver role, who attends all the patient needs, it's acquired by the father or mother of the minor. The demands of that labor can bring negative physiological and psychosocial consequences. Objective: to determine the relationship and the difference by sex and disease phase of the minor in the levels of stress, burden and anxiety of primary caregivers of boys and girls with leukemia in Hidalgo. Method: through a non-probability sampling of volunteers subjects were selected $\mathrm{N}=$ 50 female primary caregivers. To measure the variables they were used the following instruments: Perceived Stress Scale, Zarit Caregiver Burden Scale and Beck Anxiety Inventory. Results: showed positive and statistically significant correlations between anxiety-burden and stress-anxiety. It also found that the caregivers of boys have higher levels of anxiety than caregivers of girls and caregivers of patients in active phase of the disease have higher burden compared with caregivers of patients in remission phase. Conclusion: there are some high levels of stress, burden and anxiety in caregivers. There exists correlation between anxiety-stress and burdenanxiety. The caregivers of boys have more anxiety. The caregivers of children in active phase of the disease present more burden.
\end{abstract}

Keywords: Stress, burden, anxiety, primary caregiver 


\section{Resumen}

La leucemia es el tipo de cáncer que más se presenta en niños y adolescentes. La naturaleza crónica-degenerativa de la enfermedad hace que los menores necesiten de alguien responsable de su cuidado conocido como cuidador primario informal. Generalmente, el rol de cuidador primario, quien atiende todas las necesidades del paciente, es adquirido por el padre o madre del menor. Las demandas de dicha labor pueden traer consecuencias negativas a nivel fisiológico y psicosocial. Objetivo: determinar la relación y la diferencia por sexo y fase de la enfermedad del menor en los niveles de estrés, sobrecarga y ansiedad en cuidadoras primarias de niños y niñas con leucemia en Hidalgo. Método: a través de una muestra no probabilística de sujetos voluntarios se seleccionaron $\mathrm{N}=50$ cuidadoras primarias. Para medir las variables se utilizaron los siguientes instrumentos: Escala de Estrés Percibido, Escala de Carga del Cuidador de Zarit e Inventario de Ansiedad de Beck. Resultados: se encontraron correlaciones positivas y estadísticamente significativas entre ansiedad-sobrecarga y estrés-ansiedad. También se encontró que las cuidadoras de niños presentan mayores niveles de ansiedad que las cuidadoras de niñas y las cuidadoras de pacientes en fase activa de la enfermedad presentan mayor sobrecarga en comparación con las cuidadoras de pacientes en fase de remisión. Conclusión: hay algunos niveles altos de estrés, sobrecarga y ansiedad en las cuidadoras. Existe correlación entre ansiedad-estrés y sobrecarga-ansiedad. Las cuidadoras de niños presentan más ansiedad. Las cuidadoras de menores en fase activa de la enfermedad presentan mayor sobrecarga.

Palabras-claves: Estrés, sobrecarga, ansiedad, cuidador primario

\section{Introduction}

En los últimos años el número de personas con cáncer ha ido creciendo a nivel mundial, esto debido a las tasas de incidencia y de supervivencia, convirtiéndose así, en un problema grave de salud (International Agency for Research on Cancer [IARC], 2016). La leucemia es el tipo de neoplasia que más se presenta en niños y adolescentes, y se define como un tipo de cáncer que se origina en las células sanguíneas primitivas de la médula ósea (American Cancer Society [ACS], 2015). Esta enfermedad es producida por un crecimiento inocontrolado de blastos, mismos que se acumularán en la médula ósea logrando desplazar a las células sanas (Grau, 2002; National Cancer Institute [NCI], 2016).

Cabe mencionar que de todos los casos de cáncer que son diagnosticados, sólo entre el $0.5 \%$ y el $4.6 \%$ corresponde a población pediátrica (Organización Mundial de la Salud [OMS], 2016), siendo la 
leucemia la patología que más se presenta en esta etapa (ACS, 2016). En México, del 2008 al 2014, el 43\% del total de cánceres en menores de 18 años fueron por leucemia (Secretaría de Salud [SSa], 2014); y específicamente en Hidalgo, el Desarrollo Integral de la Familia (DIF, 2015) a través del Hospital del Niño atiende 125 casos de este tipo de cáncer.

Debido a los avances científicos el cáncer se ha convertido en una enfermedad crónico-degenerativa, a comparación de años atrás donde era vista como una enfermedad fatal. Este cambio en la condición del cáncer ha requerido que al mismo tiempo aumente el número de cuidadores informales (Masa'Deh, Collier, \& Hall, 2012).

De manera general, la American Society of Clinical Oncology (ASCO, 2015) define al cuidador (usualmente un familiar o amigo) como la persona que provee de apoyo físico, práctico y emocional a una persona enferma. A partir de esta de esta definición general, se han identificado dos tipos de cuidadores, formales e informales, recalcando que ambos se interrelacionan y comparten el carácter directo y prolongado de la atención al paciente (Ruíz \& Nava, 2012).

Gómez-Martinho (2016) describe al cuidador formal como un profesional cuyo trabajo es especializado, y puede ser brindado en instituciones de salud o por vía privada. Es importante mencionar que este tipo de cuidador tiene tres características principales: su trabajo es remunerado económicamente, tienen límite de tiempo para la atención, así como menor compromiso afectivo hacia el paciente (Ruíz \& Nava, 2012).

Por otro lado, el cuidador informal es aquel que brinda cuidado y atención a familiares o personas con las que tiene un lazo emocionalafectivo, dicha labor es brindada a personas que tienen algún grado de discapacidad o dependencia (Gómez-Martinho, 2016). Dentro de sus actividades están el proveer apoyo emocional, dar medicamentos y ayudar en quehaceres domésticos, siendo el cuidador primario quien tiene mayores responsabilidades (ASCO, 2015).

Ballestas et al. (2013) afirman que en los niños/niñas que padecen cáncer los cuidadores primarios son principalmente los padres o madres, de los cuales generalmente es esta última quien asume el rol.

Los tipos de cuidados que requiere un menor con diagnóstico de leucemia son actividades de autocuidado las cuales el cuidador o cuidarora realizan por él, algunas de ellas son levantar al paciente de la cama, bañarlo, asearlo, vestirlo y darle de comer. Este cuidador también cumple con algunas actividades intrumentales derivadas del cuidado como: preparar alimentos, transportarlo, gestionar finanzas y dar medicinas. A la par el cuidador o cuidadora requiere seguir cubriendo los roles que tenía antes de cuidar al menor con leucemia los cuáles suelen ser su rol familiar, gestionar finanzas, 
cuidar a otros hijos, darse tiempo con su pareja, entre otras (Ruíz \& Nava, 2012).

Cuando se habla de las consecuencias que ocasiona el cáncer, generalmente se centra en ver cómo repercuten en el enfermo, sin embargo, no se deben minimizar las repercusiones que hay en las personas que rodean al paciente con cáncer y en particular en lo que le ocurre al cuidador primario, ya que la gran función que éste desempeña puede ocasionarle tanto problemas de salud físicos como problemas de salud mental, afectando diversas áreas de su vida (Barrón \& Alvarado, 2009).

Frecuentemente los cuidadores primarios tienen la sensación de que a partir de la ejecución de este rol su salud ha empeorado, y de hecho, la salud de los padres y madres de un menor con algún tipo de padecimiento o necesidad especial se ve mayormente afectada en comparación de padres con hijos saludables. Estos problemas de salud son debidos a las necesidades que se deben cubrir en el menor (Bekenkamp, Klasina, Blomers, \& Tomic, 2014).

De manera general, el cuidador primario puede experimentar dolores de cabeza o migrañas, dolores en otras partes del cuerpo, patologías ostearticulares y musculares, problemas gástricos o dolencias estomacales, alteraciones del apetito y del peso, temblor fino, sensación de falta de aire o ahogo, arritmias, palpitaciones, sudoraciones, vértigo, alergias inmotivadas, trastornos del sueño (insomnio/sueño no reparador) o alteración de su ciclo, cansancio o agotamiento físico, fatiga crónica y sobrecarga (Espín, 2008; Romero, 2008; Ruíz \& Nava, 2012).

A nivel psicológico las consecuencias más comunes en el cuidador primario son: miedo, preocupación, desesperación, frustración, enojo/ira, irritabilidad, resentimiento, vergüenza, sentimiento de inutilidad e indefensión, culpa, nerviosismo, angustia, tensión, agotamiento mental, ansiedad o trastornos de ansiedad, sobrecarga, estrés, depresión o síntomas depresivos (tristeza, deseos de llorar, pesimismo, apatía, desesperanza), labilidad emocional, fallas en la memoria, ideas obsesivas/paranoides o suicidas, hipocondría o síntomas hipocondríacos y reacciones fóbicas (Espín, 2008; Romero, 2008; Ruíz \& Nava, 2012).

Por otra lado, a nivel familiar, el cuidador primario puede tener conflictos o deterioro en sus relaciones con los otros miembros de la familia. Esto se debe al desacuerdo acerca de la manera en que el cuidador brinda los cuidados o porque esta figura percibe que el resto de la familia no aprecia su esfuerzo o no lo apoya. Además, como la atención se centra en el enfermo se puede llegar al descuido de los otros integrantes del núcleo familiar (Espín, 2008; Ruíz \& Nava, 2012).

Laboralmente hablando, puede haber reducción de la jornada laboral, dificultades en el trabajo o pérdida total de éste (Romero, 2008). 
Directamente relacionado, Ruíz y Nava (2012) explican que en cuanto a la economía también pueden existir ciertas dificultades causadas por dos razones principales: la disminución de ingresos (debido a la disminución laboral) o el aumento de gastos como consecuencia de los cuidados.

Como se puede observar, hay diversas variables que afectan la calidad de vida del cuidador primario, sin embargo, en este estudio se decidió evaluar el estrés, la sobrecarga y la ansiedad, ya que aunque la investigación ha ido creciendo aún hay diversos factores que se desconocen sobre estas variables en dicha población.

En la actualidad, estrés es un término común, pero al mismo tiempo complejo, ya que pese a todas las conceptualizaciones que se han hecho, todavía no hay un concenso en su definición (García, 2011). Un factor que influye en esta diversidad, es que el estrés puede explicarse a través de tres modelos principales: focalizado en la respuesta, propuesto por Selye (1936); estrés como estímulo, propuesto por Holmes y Rahe (1967); y estrés como valoración cognitiva, propuesto por Lazarus y Folkman $(1984,1986)$.

Este último modelo también es conocido como Teoría basada en la interacción, desde la cual el estrés es definido como el proceso mediadortransaccional entre sujeto y ambiente. Dentro de este sistema se destacan dos actividades cognitivas fundamentales: la evaluación cognitiva $y$ el afrontamiento (Lazarus \& Folkman, 1984).

El impacto o reacción ante un acontecimiento en particular, dependerá primeramente de cómo la evalúe el sujeto, ya que esta puede ser percibida como amenzante o irrelevante. Además, también dependerá de la evaluación que haga la persona acerca de sus propias habilidades o recursos con los que cuenta para hacer frente a dicha situación (Lazarus \& Folkman, 1984).

De acuerdo a la American Psychological Association (APA, 2015) el estrés se puede dividir en tres tipos de acuerdo a su duración: el estrés agudo surge de las presiones/demandas del pasado reciente y/o del futuro cercano y sus síntomas son fáciles de reconocer. Por otro lado, el estrés agudo episódico se presenta debido a la agitación prolongada producida por un estilo de vida con desorden, apuración y demasiadas responsabilidades. Y finalmente, el estrés crónico resulta agotador y desgastante ya que está presente día con día durante un tiempo bastante prolongado causando estragos a largo plazo similares al estrés agudo, sólo que en este caso se presentan de manera constante y persistente.

Directamente relacionado con esta investigación, hay algunos estudios que reportan la presencia de estrés en personas que se dedican al cuidado de menores con cáncer. Uno de estos estudios es el realizado en Holanda por Sulkers et al. (2015), el cual reportó que las mamás solteras y las mamás con un solo hijo que fungían como cuidadoras primarias de 
ñiños/niñas con diagnóstico de cáncer, destacaron con los puntajes más altos de esta variable.

Por otro lado, Masa'Deh et al. (2012) en su estudio realizado en Jordania, encontraron que los padres cuidadores primarios y las madres cuidadoras primarias de menores con cáncer presentaban altos puntajes de estrés.

Otra de las variables de esta investigación es la sobrecarga, que se entiende como un "estado psicológico que resulta de la combinación de trabajo físico, presión emocional, restricciones sociales, así como demandas económicas que surgen al cuidar un enfermo crónico o con discapacidad" (Zarit, 2002, p. 396).

Según Barrón y Alvarado (2009) a nivel físico el cuidador puede tener las siguientes repercusiones: cansancio, dolor de cabeza, dispepsia, vértigo, dificultades para dormir y dolores articulares.

El impacto en la salud de los cuidadores de personas con cáncer en comparación con cuidadores de personas con otros padecimientos es diferente, ya que se ha demostrado que cuidadores de personas con cáncer tienen mayores niveles de sobrecarga (Ortega-Ortega, Montero-Granados, \& Jiménez-Aguilera, 2017). Además, una mayor presencia de sobrecarga se relaciona con el grado de dependencia de la persona a la que se cuida, ya que a mayor grado de dependencia mayores niveles de sobrecarga, provocando así un sentimiento de agobio (Gómez-Martinho, 2016).

Un estudio realizado en Chile concluye que los cuidadores no presentaron sobrecarga, sólo aquellos que cuidaban de personas totalmente dependientes (Caqueo-Urízar, Segovia-Lagos, Urrutia-Urrutia, Miranda, \& Navarro, 2013).

Por su parte, Rubira, Munhoz, Martinez, Barbosa y Silva (2011) afirman que el dolor, el aspecto físico, los gastos extra, el tener que faltar al trabajo y las exigencias del niño son variables que influyeron en la sobrecarga de su muestra.

Por último, Montero, Jurado y Méndez (2015) realizaron un estudio en donde encontraron que la mayor parte de su muestra presenta un nivel de carga, 90 de los 100 participantes reportaron afectaciones en una o más áreas de su vida (trabajo, escuela, tareas del hogar, vida social, vida familiar, vida sexual, pasatiempos, vacaciones y convivencia con la pareja), concluyendo que existe una correlación positiva entre el número de áreas afectadas en la vida del cuidador primario y la carga que experimenta.

La última variable de estudio de esta investigación es la ansiedad, definida como un estado emocional subjetivamente desagradable caracterizado por sentimientos molestos tales como tensión o nerviosismo, y síntomas fisiológicos como palpitaciones cardíacas, temblor, náuseas y vértigo (Beck, Epstein, Brown, \& Steer, 1988). 
En el caso del cuidado, Gómez-Martinho (2016) menciona que en ocasiones los altos niveles de ansiedad se relacionan con el desconocimiento de cómo se debe desempeñar la labor de cuidado, provocando así la elevación de ansiedad.

Al igual que el estrés, hay diversos estudios que reportan la presencia de ansiedad en cuiadores primarios y cuidadoras primarias de personas con cáncer.

Kohlsdorf y Costa (2011) realizan un estudio en donde encuentran que las cuidadoras y los cuidadores de menores con leucemia de su muestra presentan ansiedad moderada, lo que en el futuro se pudiese agravar. Por otra parte, en el estudio de dos Santos, Brito y Yamaguchi (2013) realizado en Brasil, se llegó a la conclusión que los niveles de ansiedad en padres y madres de menores con cáncer, estuvieron directamente correlacionados con la edad del niño, el tiempo de diagnóstico, el tiempo de tratamiento, la edad de los padres y la renta familiar.

Más específicamente, no se tiene muy claro si existen diferencias entre las cuidadoras de niños y niñas con respecto al nivel de ansiedad, pero si se sabe que la fase de la enfermedad en que se encuentre el menor influirá en la sobrecarga del cuidador (Northouse, Katapodi, Schafenacker, \& Weiss, 2012).

Por otro lado, el estudio de McDaniel y Allen (2012), llega a la conclusión de que existe una relación positiva y estadísticamente significativa entre las variables de estrés y sobrecarga. Concluyendo además, que un factor que predice la presencia de ambas variables es el empleo, es decir, si los cuidadores y cuidadoras trabajan o no. Otro estudio similar es el de González de Mirena et al. (2015) realizado en Venezuela, el cual concluyó que hubo una alta y significativa relación entre la sobrecarga y todos los indicadores del estrés, sobre todo en el somático, lo que quiere decir que el cuidador tiende a somatizar el problema.

En cuanto a la relación entre ansiedad y estrés, el estudio de PedrónGiner, Calderón, Martínez-Costa, Borraz-Gracia y Gómez-López (2012), reporta que la mayoría de su muestra presenta alto riesgo de padecer síntomas de ansiedad, mismos que son influenciados por el estrés diario. Además, Sulkers et al. (2015), llegan a la conclusión de que un mayor nivel de estrés está asociado a un mayor nivel de ansiedad.

Finalmente, el estudio de Montero et al. (2015), además de evaluar el nivel de sobrecarga y ansiedad, concluye que hay una correlación positiva y estadísticamente significativa entre carga y ansiedad, concluyendo que ésta es una predictora de la carga global del cuidador.

El propósito de la siguiente investigación fue determinar la relación y la diferencia por sexo y fase de la enfermedad del menor en los niveles de 
estrés, sobrecarga y ansiedad en cuidadoras primarias de niños que padecen leucemia en Hidalgo.

Como hipótesis acerca de correlación se plantea que sí existe relación estadísticamente significativa entre el nivel de estrés, el nivel de ansiedad y el nivel de sobrecarga en cuidadoras primarias de niños con leucemia de Hidalgo.

Por otro lado, como hipótesis sobre diferencias se plantea que sí existe diferencia por sexo y fase de la enfermedad en el nivel de estrés, el nivel de ansiedad y el nivel de sobrecarga en cuidadoras primarias de niños con leucemia de Hidalgo.

\section{Método}

\section{Participantes}

Participaron 50 cuidadoras primarias de niños con diagnóstico confirmado de leucemia, usuarios de la Asociación Mexicana de Ayuda a Niños con Cáncer (AMANC) Hidalgo. Dichas mujeres fueron seleccionadas mediante un muestreo no probabilístico, accidental por cuota. El rango de edad osciló entre los 24 y 67 años, con un promedio de 40.20 años y una desviación estándar de 10.14.

Tabla 1.Datos sociodemográficos de las cuidadoras.

\begin{tabular}{ccc}
\hline & Frecuencia & Porcentaje \% \\
\hline Estado civil & 9 & 18 \\
Soltera & 21 & 42 \\
Casada & 20 & 40 \\
Otro & & \\
Nivel educacional & 19 & 38 \\
Primaria & 19 & 38 \\
Secundaria & 9 & 18 \\
Bachillerato & 3 & 6 \\
Licenciatura & & \\
Situación de empleo & & 34 \\
formal & 17 & 66 \\
Sí & 33 & \\
No & &
\end{tabular}

\section{Instrumentos}

Escala de Estrés Percibido: se utilizó la Escala de Estrés Percibido (PSS) de Cohen, Kamarck y Mermelstein (1983), validada en México por González y Landero (2007). Dicha escala consta de 14 reactivos divididos en dos factores que son: control de estrés y falta de control de estrés. Cuenta con un alfa de Cronbach de 0.83 y tiene un formato de respuesta tipo Likert con cinco opciones que son: 0 (nunca), 1 (casi nunca), 2 (de vez en cuando), 3 (a menudo) y 4 (muy a menudo). 
Tiene un punto de corte de 30 propuesto por Moral, González y Landero (2011), donde arriba o igual a este puntaje significa que hay niveles de estrés alto y por debajo del punto de corte indica que hay niveles de estrés bajo.

Escala de Carga del Cuidador de Zarit: se utilizó la Escala de Carga del Cuidador de Zarit (Zarit, Reever, \& Bach-Peterson, 1980), validada en México por Montero, Jurado, Valencia, Méndez y Mora (2014). Consta de 12 reactivos divididos en tres factores que son: impacto del cuidado, relación interpersonal y expectativas de autoeficacia. Cuenta con un alfa de Cronbach de 0.84 y tiene un formato de respuesta tipo Likert con cinco opciones que son: 1 (nunca), 2 (casi nunca), 3 (algunas veces), 4 (frecuentemente) y 5 (siempre). Sus puntos de corte son: de 12 a 22 hay ausencia de carga, de 23 a 29 carga leve y de 30 a 60 carga excesiva.

Inventario de Ansiedad de Beck: se utilizó el Inventario de Ansiedad de Beck (Beck et al., 1988), validado en México por Robles, Varela, Jurado y Páez (2001). Este instrumento consta de 21 reactivos con un alfa de Cronbach de 0.83 y tiene un formato de respuesta tipo Likert con cuatro opciones que son: 0 (poco o nada), 1 (más o menos), 2 (moderadamente) y 3 (severamente). Sus puntos de corte son: de 0 a 5 ansiedad mínima, de 6 a 15 ansiedad leve, de 16 a 30 ansiedad moderada y de 31 a 63 ansiedad severa.

\section{Procedimiento}

Se aplicaron de manera individual y voluntaria todos losinstrumentos en un solo momento a las participantes dentro de las instalaciones de AMANC Hidalgo. Posteriormente, se llevaron acabo análisis de medidas de tendencia central, el coeficiente de correlación de Pearson y la prueba $t$ de student para muestras independientes.

\section{Resultados}

El objetivo general de este estudio fue determinar la relación y la diferencia por sexo y fase de la enfermedad del menor en los niveles de estrés, sobrecarga y ansiedad en cuidadoras primarias de niños que padecen leucemia en Hidalgo.

En la tabla 2 se describen las medidas de tendencia central y dispersión de la variable estrés, donde se puede observar que la media del puntaje general es de 27.42 mismo que se encuentra por debajo del punto de corte (30) propuesto por Moral et al. (2011), lo que quiere decir que en general las cuidadoras primarias no presentan niveles considerables de estrés. 
Tabla 2. Medidas de tendencia central y dispersión de los factores de la variable estrés.

\begin{tabular}{cccc}
\hline Variable & Rango Teórico & Media & Desviación Estándar \\
\hline Estrés & & & \\
\hline Control de estrés & $0-28$ & 16.32 & 5.82 \\
Falta de control de & $0-28$ & 15.74 & 5.70 \\
estrés & $0-56$ & 27.42 & 8.09 \\
Puntaje general &
\end{tabular}

Continuando con los resultados de esta variable, la tabla 3 muestra la distribución del nivel de estrés en las cuidadoras de esta investigación.

Tabla 3. Distribución del nivel de estrés en las cuidadoras.

\begin{tabular}{ccc}
\hline Nivel de estrés & Número de cuidadoras & Porcentaje $\%$ \\
\hline Estrés bajo & 25 & 50 \\
Estrés alto & 25 & 50 \\
\hline
\end{tabular}

En la tabla 4 se describen las medidas de tendencia central y dispersión de la variable sobrecarga, donde se puede observar que la media más alta se encontró en el factor impacto del cuidado con un puntaje de 12.38, mientras que la media más baja la tuvo el factor expectativas de autoeficacia con 7.96. En base al resultado obtenido en la media del puntaje general de sobrecarga (31.22) se concluye que las cuidadoras presentan sobrecarga excesiva.

Tabla 4. Medidas de tendencia central y dispersión de los factores de la variable sobrecarga.

\begin{tabular}{cccc}
\hline Variable & Rango Teórico & Media & Desviación Estándar \\
\hline Sobrecarga & & & \\
\hline Impacto del cuidado & $5-25$ & 12.38 & 5.52 \\
$\quad$ Relación & $5-25$ & 10.88 & 5.28 \\
interpersonal & & 7.96 & 2.32 \\
Expectativas de & $2-10$ & $31 . .22$ & 11.24 \\
$\quad$ autoeficacia & $12-60$ &
\end{tabular}

Continuando con los resultados de esta variable, la tabla 5 muestra la distribución del nivel de sobrecarga en las cuidadoras de esta investigación.

Tabla 5. Distribución del nivel de sobrecarga en las cuidadoras.

\begin{tabular}{ccc}
\hline Nivel de sobrecarga & Número de cuidadoras & Porcentaje $\%$ \\
\hline Ausencia de carga & 19 & 38 \\
Carga leve & 9 & 18 \\
Carga excesiva & 22 & 44 \\
\hline
\end{tabular}

En la tabla 6 se describen las medidas de tendencia central y dispersión de la variable ansiedad, donde se puede observar que la media más alta se encontró en el factor subjetivo con un puntaje de 10.40. En base al resultado obtenido en la media del puntaje general de ansiedad (21.94) se concluye que las cuidadoras presentan ansiedad moderada. 
Tabla 6. Medidas de tendencia central y dispersión de los factores de la varibale ansiedad.

\begin{tabular}{cccc}
\hline Variable & Rango Teórico & Media & Desviación Estándar \\
\hline Ansiedad & & & \\
\hline Subjetivo & $0-24$ & 10.40 & 5.66 \\
Neurofísiológico & $0-21$ & 6.60 & 5.42 \\
Autonómico & $0-9$ & 2.46 & 2.46 \\
Pánico & $0-9$ & 2.48 & 1.76 \\
Puntaje general & $0-63$ & 21.94 & 13.50 \\
\hline
\end{tabular}

Continuando con los resultados de esta variable, la tabla 7 muestra la distribución del nivel de ansiedad en las cuidadoras de esta investigación.

Tabla 7. Distribución del nivel de ansiedad en las cuidadoras.

\begin{tabular}{ccc}
\hline Nivel de ansiedad & Número de cuidadoras & Porcentaje $\%$ \\
\hline Ansiedad mínima & 6 & 12 \\
Ansiedad leve & 13 & 26 \\
Ansiedad moderada & 16 & 32 \\
Ansiedad severa & 15 & 30 \\
\hline
\end{tabular}

Para determinar la diferencia por sexo del menor en el nivel de estrés, sobrecarga y ansiedad en cuidadoras primarias de niños que padecen leucemia en Hidalgo, se utilizó la prueba $t$ de student para muestras independientes, obteniéndose resultados significativos sólo en la variable de ansiedad.

La tabla 8 muestra los resultados de las diferencias por sexo del menor en el nivel de ansiedad de las cuidadoras de niños y niñas con leucemia. Se puede observar que se obtuvieron diferencias estadísticamente significativas en los factores neurofisiológico $t(43.84)=2.08 p<.05$; en el factor autonómico $t(48)=2.04 p<.05$; en el factor pánico $t(47.02)=2.30 p<.05$, y en el puntaje de ansiedad total $t(45.53)=2.30 p<.05$, siendo las cuidadoras de los niños quienes presentan un mayor puntaje a comparación de las cuidadoras de las niñas. No se encontraron diferencias estadísticamente significativas en el nivel de estrés y sobrecarga de cuidadoras de niños y niñas con leucemia.

Tabla 8. Comparaciones por sexo del menor de la variable ansiedad.

\begin{tabular}{ccccccc}
\hline \multicolumn{2}{c}{ Niños } & \multicolumn{7}{c}{ Niñas } \\
\hline Factores & Media & D.E. & Media & D.E. & $t$ & $P$ \\
\hline Neurofisiológico & 7.89 & 6.30 & 4.95 & 3.52 & 2.08 & $.043^{*}$ \\
Autonómico & 3.07 & 2.56 & 1.68 & 2.12 & 2.04 & $.046^{*}$ \\
Subjetivo & 11.64 & 6.11 & 8.82 & 4.70 & 1.84 & .071 \\
Pánico & 2.89 & 1.96 & 1.95 & 1.32 & 2.00 & $.05^{*}$ \\
Ansiedad total & 22.50 & 15.27 & 17.41 & 9.32 & 2.30 & .026 \\
\hline \multicolumn{7}{c}{$* 0.05$}
\end{tabular}

De igual manera, para determinar la diferencia por fase de la enfermedad del menor en los niveles de estrés, sobrecarga y ansiedad en cuidadoras primarias de niños que padecen leucemia en Hidalgo, se utilizó la 
prueba $t$ de student para muestras independientes, obteniéndose resultados significativos sólo en la variable de sobrecarga.

En la tabla 9 se muestran los resultados de las diferencias por fase de la enfermedad del menor en el nivel de sobrecarga de las cuidadoras de niños y niñas con leucemia. Se puede observar que hay diferencias estadísticamente significativas en los factores impacto del cuidado $t(48)=2.40 p<.05$; en el factor relación interpersonal $t(48)=2.36 p<.05$; y en el nivel de sobrecarga total $t(48)=2.25 p<.05$, siendo las cuidadoras de los menores en fase activa de la enfermedad quienes presentan un mayor puntaje en comparación con las cuidadoras de los menores en fase de remisión. No se encontraron diferencias estadísticamente significativas en el nivel de estrés y ansiedad de las cuidadoras en estas dos fases de la enfermedad.

Tabla 9. Comparaciones por fase de la enfermedad del menor de la variable sobrecarga.

\begin{tabular}{|c|c|c|c|c|c|c|}
\hline & \multicolumn{2}{|c|}{ Fase activa } & \multicolumn{2}{|c|}{ Fase de remisión } & \multirow[b]{2}{*}{$t$} & \multirow[b]{2}{*}{$P$} \\
\hline Factores & Media & D.E. & Media & D.E. & & \\
\hline $\begin{array}{l}\text { Impacto del } \\
\text { cuidado }\end{array}$ & 13.67 & 5.46 & 9.88 & 5.11 & 2.40 & $.020 *$ \\
\hline $\begin{array}{c}\text { Relación } \\
\text { interpersonal }\end{array}$ & 12.09 & 5.17 & 8.53 & 4.79 & 2.36 & $.022 *$ \\
\hline $\begin{array}{l}\text { Expectativas de } \\
\text { autoeficacia }\end{array}$ & 7.94 & 2.38 & 8.00 & 2.26 & -0.87 & .093 \\
\hline Sobrecarga total & 33.70 & 11.24 & 26.41 & 9.86 & 2.25 & $.028 *$ \\
\hline
\end{tabular}

Para determinar la relación que existe entre el nivel de estrés y el nivel de sobrecarga en cuidadoras primarias de niños que padecen leucemia en Hidalgo, se utilizó una correlación de Pearson.

En la tabla 10 se puede observar que existe una correlación positiva y estadísticamente significativa entre el factor impacto del cuidado de la Escala de Carga del Cuidador con el factor falta de control de estrés de la Escala de Estrés Percibido. Esta correlación se mantiene entre el factor relación interpersonal de la ECC con el factor falta de control de estrés de la EPP. Por otro lado, no se presenta correlación estadísticamente significativa entre el factor control de estrés de la EEP y alguno de los factores de la ECC; al igual que entre el factor expectativas de autoeficacia de la ECC y falta de control de estrés de la EEP.

Por último, tomando en cuenta los puntajes generales de ambas escalas, se concluye que no existe una correlación estadísticamente significativa entre el nivel de estrés percibido y el nivel de sobrecarga en las cuidadoras de esta investigación.

Tabla 10. Relación entre estrés y sobrecarga.

Falta de control de estrés
Estrés

Control de estrés
Estrés total 


\begin{tabular}{cccc} 
Impacto del cuidado & $.437^{* *}$ & .085 & .246 \\
Relación interpersonal & $.461^{* *}$ & .049 & $.289^{*}$ \\
Expectativas de autoeficacia & .212 & .088 & .086 \\
Sobrecarga total & $.475^{* *}$ & .083 & .275 \\
\hline
\end{tabular}

**.La correlación es significativa en el nivel 0,01 (bilateral).

*. La correlación es significativa en el nivel 0,05 (bilateral).

De igual manera, para determinar la relación que existe entre el nivel de estrés y el nivel de ansiedad en cuidadoras primarias de niños que padecen leucemia en Hidalgo, se utilizó una correlación de Pearson.

En la tabla 11 se puede apreciar que entre el factor falta de control de estrés de la Escala de Estrés Percibido y todos los factores del Inventario de Ansiedad de Beck existe correlación positiva y estadísticamente significativa. Respecto al factor control de estrés de la EEP existe una correlación negativa pero no estadísticamente significativa con la mayoría de los factores correspondientes al IAB, esto puede deberse a que los síntomas de ansiedad no compaginan con el cómo una persona controla su estrés y por lo tanto al controlar su estrés no presentan algún tipo de sintomatología.

Por último, tomando en cuenta los puntajes generales de ambas escalas, se concluye que existe una correlación positiva y estadísticamente significativa entre el nivel de estrés percibido y el nivel de ansiedad en las cuidadoras de esta investigación.

Tabla 11. Relación entre estrés y ansiedad.

\begin{tabular}{cccc}
\hline & \multicolumn{3}{c}{ Estrés } \\
\hline Ansiedad & Falta de control de & Control de & Estrés total \\
Subjetivo & estrés & estrés & $.359^{*}$ \\
Neurofisiológico & $.421^{* *}$ & -.087 & $.449^{* *}$ \\
Autonómico & $.514^{* *}$ & -.120 & $.502^{* *}$ \\
Pánico & $.506^{* *}$ & -.203 & $.303^{*}$ \\
Ansiedad total & $.475^{* *}$ & 0.44 & $.462^{* *}$ \\
\hline
\end{tabular}

**.La correlación es significativa en el nivel 0,01 (bilateral).

*. La correlación es significativa en el nivel 0,05 (bilateral).

Por último, para determinar la relación que existe entre el nivel de estrés y el nivel de sobrecarga en cuidadoras primarias de niños que padecen leucemia en Hidalgo, se utilizó una correlación de Pearson.

En la tabla 12 se puede apreciar que los factores impacto del cuidado y relación interpersonal de la Escala de Carga del Cuidador tienen correlación positiva y estadísticamente significativa con todos los factores del Inventario de Ansiedad de Beck. En cuanto al factor expectativas de autoeficacia no se presenta alguna relación estadísticamente significativa con alguno de los factores del IAB.

Tomando en cuenta los puntajes generales de ambas escalas, se concluye que existe una correlación positiva y estadísticamente significativa 
entre el nivel de sobrecarga y el nivel de ansiedad en las cuidadoras de esta investigación.

Tabla 12. Relación entre sobrecarga y ansiedad.

\begin{tabular}{ccccc}
\hline \multicolumn{4}{c}{ Sobrecarga } \\
\hline Ansiedad & $\begin{array}{c}\text { Impacto del } \\
\text { cuidado }\end{array}$ & $\begin{array}{c}\text { Relación } \\
\text { interpersonal }\end{array}$ & $\begin{array}{c}\text { Expectativas } \\
\text { de } \\
\text { autoeficacia }\end{array}$ & $\begin{array}{c}\text { Sobrecarga } \\
\text { total }\end{array}$ \\
Subjetivo & $.688^{* *}$ & $.627^{* *}$ & .135 & $.661^{* *}$ \\
Neurofisiológico & $.636^{* *}$ & $.620^{* *}$ & .017 & $.607 * *$ \\
Autonómico & $.520^{* *}$ & $.516^{* *}$ & -.007 & $.497^{* *}$ \\
Pánico & $.372^{* *}$ & $.330^{* *}$ & .095 & $.358^{*}$ \\
Ansiedad total & $.687^{* *}$ & $.650^{* *}$ & .074 & $.658^{* *}$ \\
\hline$* *$ La correlación es significativa en el nivel 0,01 (bilateral). \\
*. La correlación es significativa en el nivel 0,05 (bilateral).
\end{tabular}

\section{Conclusión}

El perfil que se obtuvo de las cuidadoras de esta investigación es el siguiente: son mujeres casadas con un promedio de edad de 40.20 años, con educación básica, no trabajan, dependen económicamente de su familia o pareja y tienen un promedio de 3.08 hijos. En total son 28 niños y 22 niñas, y del total de la muestra 33 menores están en fase activa de la enfermedad y 17 en fase de remisión con un promedio de edad de 10.20 años.

En cuanto a estrés, la mitad de la muestra se ubica por arriba del punto de corte de la prueba, es decir presentan niveles de estrés alto, mientras que la otra mitad no.

Estos resultados concuerdan con el estudio de Masa'Deh et al. (2012), donde se encontró que tanto padres cuidadores primarios como madres cuidadoras primarias de menores con cáncer presentaban altos puntajes de estrés.

El estudio longitudinal de Sulkers et al. (2015) realizado en Holanda, también reportó resultados similares, encontrando que las mamás solteras y las mamás con un solo hijo que fungían como cuidadoras primarias, destacaron con los puntajes más altos de estrés. En esta investigación, se puede observar que la submuestra de cuidadoras que tienen un solo hijo presentan altos niveles de estrés, mientras que las que tienen más de un hijo reportan estar equilibradas. Patterson, Holm y Gurney (2004) explican que a pesar de que tener más hijos implica una carga, por otra parte esto permite que las madres tengan un apoyo extra y al mismo tiempo éstos pueden servir como distracción para la cuidadora.

En cuanto al nivel de sobrecarga, más de la mitad de las cuidadoras presentan algún nivel de sobrecarga y de estas, la mayoría presentan carga excesiva.

Los resultados de Montero et al. (2015) se acercan a los de esta investigación ya que al menos el $74 \%$ de su muestra obtuvo algún nivel de 
sobrecarga, el $46 \%$ tuvo carga leve y el $28 \%$ carga excesiva. Además, las medias en cuanto al tiempo de cuidado de ambos estudios no difieren mucho. Krikorian, Vélez, González, Palacio y Vargas (2010) postulan que la presencia de algún grado de sobrecarga guarda relación con el tiempo de cuidado.

Por otro lado, estos resultados difieren de los encontrados en el estudio de Caqueo-Urízar et al. (2013) donde de manera general la muestra no experimentó sobrecarga. Cabe mencionar que el estudio anterior se realizó en cuidadores primarios de adultos con cáncer, mientras que en esta investigación, la población oncológica fueron menores de edad. La diferencia en los resultados se explica por el vínculo existente entre los cuidadores/cuidadoras con el paciente enfermo, ya que según Carretero, Garcés y Ródenas (2006) si el cuidador es cónyugue o padre/madre se generan mayores índices de sobrecarga.

Por último, se encontró que en general hay ansiedad moderada en la muestra, y que más de la mitad de las cuidadoras presentan niveles moderados o severos.

Los resultados obtenidos en esta investigación concuerdan con el estudio de Grov, Dahl, Moum y Fossa (2005) donde los cuidadores primarios presentaron niveles altos de ansiedad en comparación con la media de la población general. Concluyen que la ansiedad es un indicador de probables problemas que pueden desarrollarse a futuro en el cuidador (sobrecarga, síndrome del cuidador, entre otros), generada por el impacto negativo de cuidar a un paciente enfermo (en este caso oncológico) o por temores sobre el futuro tanto del paciente como del propio cuidador primario.

Otro estudio realizado por Kohlsdorf y Costa (2011) encontró que la muestra de cuidadoras y cuidadores de menores con leucemia presentan ansiedad moderada, lo que en el futuro pudiese agravar.

Romero (2008) concluye que los estados emocionales del cuidador como lo es la ansiedad, se relacionan significativamente con dos variables que son: el deterioro funcional del enfermo y el tiempo de cuidado.

En cuanto a las diferencias por sexo del menor, se encontró que las cuidadoras de los niños presentan mayor nivel de ansiedad en comparación con las cuidadoras de las niñas. Este resultado es un hallazgo que valdría la pena investigar a mayor profundidad en estudios posteriores ya que no hay información sobre esto en la bibliografía ya existente.

Rodríguez (2006) explica que las madres tienden a enseñar de mejor manera a las niñas, a emplear el lenguaje para poder expresar sus emociones y a ser empáticas en comparación con los niños, además de que las niñas tienden a tener mayor tolerancia a estar fuera de casa que los niños, esto puede explicar los resultados obtenidos en esta investigación. Partiendo de lo anterior, es probable que las madres de niños tengan más ansiedad porque 
pueden tener mayor dificultad de enseñar a sus hijos a expresarse emocionalmente, así como enseñarles a ser empáticos, situación diferente con las madres de niñas que pueden tener mayor facilidad en enseñar lo ya mencionado.

Por otro lado, en cuanto a las diferencias por fase de la enfermedad del menor, se encontró que las cuidadoras de menores en fase activa son quienes presentan un mayor puntaje en el nivel de sobrecarga en comparación con las cuidadoras de menores en fase de remisión.

Esta diferencia se puede explicar por las fuentes de distrés emocional que existen en cada etapa de la enfermedad para el cuidador primario. Northouse et al. (2012) concluyen que la fase activa, en la cual se suministra el tratamiento, trae implicaciones negativas para el cuidador como preocupaciones por la efectividad del tratamiento, así como por las consecuencias que éste puede tener en el paciente, es decir, si el enfermo será capaz de soportar el tratamiento y los síntomas/efectos asociados a este. Por otra parte, en esta etapa el cuidador está más involucrado en proveer cuidado físico, y muchos de ellos tienen la preocupación de que su cuidado no sea óptimo. Por estas razones "no es sorprendente que durante la fase del tratamiento los cuidadores usualmente reporten mayor sobrecarga de cuidado y de estrés de lo que lo hacen en otras fases de la enfermedad" (p. 241).

Otra razón por la que la sobrecarga es mayor en esta etapa esla presencia o no de cuidadores secundarios. Como lo refleja la investigación de van Ryn et al. (2011) la mayoría de los cuidadores primarios son los únicos proveedores del cuidado del paciente y no reciben ayuda de otras personas.

En cuanto a las correlaciones entre variables, se encontró que no existe relación estadísticamente significativa entre estrés y sobrecarga en las cuidadoras primarias de esta investigación.

Es importante mencionar que el factor falta de control de estrés se correlacionó positivamente con la mayoría de los factores de sobrecarga, por lo que a mayor falta de control de estrés mayores consecuencias en el impacto del cuidado, en la relación interpersonal del cuidador y en el puntaje general de esta variable.

Estos resultados concuerdan con los encontrados en otros estudios como el de McDaniel y Allen (2012), donde se encontró que existe una relación positiva y estadísticamente significativa entre las variables antes mencionadas, concluyendo que el estrés del cuidador tiene efectos en la sobrecarga. Se concluye que una factor que predice la presencia de ambas variables es el empleo, es decir, si los cuidadores y cuidadoras trabajan o no.

El estudio de González de Mirena et al. (2015), arrojó que hubo una alta y significativa relación entre la sobrecarga y todos los indicadores del 
estrés, sobre todo en el somático, lo que quiere decir que el cuidador tiende a somatizar el problema.

Por otro lado, se encontró que existe una relación estadísticamente significativa y positiva entre la presencia de síntomas de ansiedad y la falta de control de estrés.

Estos resultados son concondartes con el estudio de Pedrón-Giner et al. (2012) donde la mayoría de su muestra presenta alto riesgo de padecer síntomas de ansiedad, mismos que son influenciados por el estrés diario.

Por último, se encontró que existe una relación estadísticamente significativa y positiva entre ansiedad y sobrecarga, ya que a mayor nivel de sobrecarga mayor nivel de ansiedad.

Ciertos estudios ya han demostrado la relación entre estas variables, por ejemplo, Pioli (2010) encontró una correlación negativa, ya que a menor nivel de sobrecarga, menor nivel de ansiedad.

Por otro lado, Montero et al. (2015) encuentran una correlación positiva de la carga con la ansiedad, concluyendo que esta última es predictora de la carga global del cuidador.

Las aportaciones del presente estudio evidencian que ser cuidadora de un menor con leucemia causa afectaciones en la calidad de vida de la antes mencionada, situación que se puede agravar a partir de diversos factores como la fase de la enfermedad en la que el menor se encuentre. Además, se debe considerar que en variables como la ansiedad se incrementarán según el sexo del menor, siendo éste, el dato más innovador y que requiere de ser investigado exhaustivamente. Por último, se logra aportar una base sólida que permitirá generar intervenciones más eficaces que propicien el mejoramiento de la calidad de vida de la cuidadora y por ende de la calidad de cuidado que brinda.

\section{References:}

1. American Cancer Society. (2015). Leucemia en niños. Recuperado de www.cancer.org/acs/groups/cid/documents/webcontent/002289pdf.pdf

2. American Cancer Society. (2016). Cancer in children. Recuperado de http://www.cancer.org/acs/groups/cid/documents/webcontent/002287 pdf.pdf

3. American Psychological Association. (2015). Los distintos tipos de estrés. Recuperado de http://www.apa.org/centrodeapoyo/tipos.aspx

4. American Society of Clinical Oncology. (2015). Being a Caregiver. Recuperado de http://www.cancer.net/coping-with-cancer/caringloved-one/being-caregiver

5. Ballestas, H., López, E., Meza, R., Palencia, K., Ramos, D., \& Montalvo, A. (2013). Cuidadores familiares de niños con cáncer y su 
funcionalidad. Salud Uninorte, 29(2), 249-259. Recuperado de http://www.redalyc.org/pdf/817/81730430010.pdf

6. Barrón, B. S., \& Alvarado, S. (2009). Desgaste físico y emocional del cuidador primario en cáncer. Cancerología, 4, 39-46. Recuperado de http://www.antesdepartir.org.mx/lecturas/Desgaste-del-cuidador.pdf

7. Beck, A. T., Epstein, N., Brown, G., \& Steer, R. A. (1988). An inventory for measuring clinical anxiety: Psychometric properties. Journal of Consulting and Clinical Psychology, 56(6), 893-897. doi: 10.1037/0022-006X.56.6.893

8. Bekenkamp, J., Klasina, H. A., Bloemers, W., \& Tomic, W. (2014). The relationship between physical health and meaning in life among parents of special needs children. Europe's Journal of Psychology, 10(1). doi:10.5964/ejop.v10i1.674

9. Caqueo-Urízar, A., Segovia-Lagos, P., Urrutia-Urrutia, U., Miranda, C., \& Navarro, E. (2013). Impacto de la relación de ayuda de cuidadores primarios en la calidad de vida de pacientes con cáncer avanzado. Psicooncología, 10(1), 95-108. doi: 10.5209/rev_PSIC.2013.v10.41950

10. Carretero, S., Garcés, J., \& Ródenas, F. (2006). La sobrecarga de las cuidadoras de personas dependientes: Análisis y propuestas de intervención psicosocial. Valencia: Tirant lo Blanch.

11. Cohen, S., Kamarck, T., \& Mermelstein, R. (1983). A global measure of perceived stress. Journal of Health and Social Behavior, 24(4), 385-396. doi: 10.2307/2136404

12. Desarrollo Integral de la Familia. (2015). Hospital del Niño DIFH atiende a 90 pacientes nuevos con cáncer, al año. Recuperado de http://dif.hidalgo.gob.mx/?p=395

13. dos Santos, D. F., de Brito, E., \& Yamaguchi, A. (2013). Estrés relacionado al cuidado: El impacto del cáncer infantil en la vida de los padres. Revista Latino-Americana de Enfermagem, 21(1), 1-7. doi: 10.1590/S010411692013000100010

14. Espín, A. M. (2008). Caracterización psicosocial de cuidadores informales de adultos mayores con demencia. Revista Cubana de Salud Pública, 34(3). Recuperado de http://www.redalyc.org/articulo.oa?id=21434302

15. García, A. (2011). Efectos del estrés percibido y las estrategias de aprendizaje cognitivas en el rendimiento académico de estudiantes universitarios noveles de ciencias de la Salud. (Tesis de Doctorado) Universidad de Málaga. España.

16. Gómez-Martinho, M. R. (2016). Cuidado formal e informal de personas mayores dependientes. Recuperado de 
https://repositorio.comillas.edu/xmlui/bitstream/handle/11531/13437/ TFM000507.pdf?sequence=1

17. González, M. T., \& Landero, R. (2007). Factor structure of the Perceived Stress Scale (PSS) in a sample from Mexico. The Spanish Journal of Psychology, 10(1), 199-206. Recuperado de http://www.psy.cmu.edu/ scohen/Factor\%20Structure_Mexico.pdf

18. González de Mirena, E., Gil, Y., Younes, T., Molina, A., Mejías, A., Rubio, A.,...Calzolaio, V. (2015). Estrés, sobrecarga e interleuquina proinflamatoria (IL1) y anti-inflamatoria (IL4) en cuidadores de pacientes oncológicos. Revista Venezolana de Endocrinología y Metabolismo, 13(2), 78-85. Recuperado de http://www.redalyc.org/articulo.oa?id=375542244003

19. Grau, C. (2002). Impacto psicosocial del cáncer infantil en la familia. Educación, Desarrollo y Diversidad, 5(2), 87-106. Recuperado de https://www.researchgate.net/publication/270889950_IMPACTO_PS ICOSO CIAL_DEL_CANCER_INFANTIL_EN_LA_FAMILIA

20. Grov, E. K., Dahl, A. A., Moum, T., \& Fossa, S. D. (2005). Anxiety, depression, and quality of life in caregivers of patients with cancer in late palliative phase. Annals of Oncology, 16(7), 1185-1191. doi: 10.1093/annonc/mdi210

21. Holmes, T. H. \& Rahe, R. H. (1967). The social readjustment ratting scale. Journal of Psychosomatic Research, 11, 213-218.

22. International Agency for Research on Cancer. (2016). GLOBOCAN 2012: Estimated cancer incidence, mortality and prevalence worldwide in 2012. Recuperado de http://globocan.iarc.fr/Default.aspx

23. Kohlsdorf, M., \& Costa, A. L. (2011). Coping strategies and caregiver's anxiety in pediatric oncohematology. Psicologia: Reflexao e Crítica, 24(2), 272-280. doi: 10.1590/S010279722011000200008

24. Krikorian, A., Vélez, M. C., González, O., Palacio, C., \& Vargas, J. J. (2010). La experiencia de sufrimiento en cuidadores principales de pacientes con dolor oncológico y no oncológico. Avances en Enfermería, 28(1), 13-20. Recuperado de http://www.scielo.org.co/pdf/aven/v28n1/v28n1a02.pdf

25. Lazarus, R. S., \& Folkman, S. (1984). Stress, appraisal and coping. New York: Springer.

26. Lazarus, R. S., \& Folkman, S. (1986). Estrés y procesos cognitivos. Barcelona: Editorial Martínez Roca.

27. Masa'Deh, R., Collier, J., \& Hall, C. (2012). Parental stress when caring for a child with cancer in Jordan: A cross-sectional survey. 
Health and Quality of Life Outcomes, 10(88), 1-7. doi: 10.1186/14777525-10-88

28. McDaniel, K. R., \& Allen, D. G. (2012). Working and care-giving: The impact on caregivers stress, family-work conflict, and burnout. Journal of Life Care Planning, 10(4), 21-32.

29. Montero, X., Jurado, S., \& Méndez, J. (2015). Variables que predicen la aparición de sobrecarga en cuidadores primarios informales de niños con cáncer. Psicooncología, 12(1), 67-86. doi: 10.5209/rev_PSIC.2015.v12.n1.48905

30. Montero, X., Jurado, S., Valencia, A., Méndez, J., \& Mora, I. (2014). Escala de carga del cuidador de Zarit: Evidencia de validez en México. Psicooncología, 11(1), 71-85.

31. Moral, J., González, M. T., \& Landero, R. (2011). Estrés percibido, ira y burnout en amas de casa mexicanas. Revista Iberoamericana de Psicología y Salud, 2(2), 123-143. Recuperado de http://www.redalyc.org/pdf/2451/245118507001.pdf

32. National Cancer Institute. (2016). Leucemia mielógena crónica: Tratamiento (PDQ®)-Versión para pacientes. Recuperado de https://www.cancer.gov/espanol/tipos/leucemia/paciente/tratamientolmcpdq

33. Northouse, L. L., Katapodi, M. C., Schafenacker, A. M., \& Weiss, D. (2012). The impact of caregiving on the psychological well-being of family caregivers and cancer patients. Seminars in Oncology Nursing, 28(4), 236-245. doi: 10.1016/j.soncn.2012.09.006

34. Organización Mundial de la Salud. (2016). Cáncer. Preguntas frecuentes sobre el cáncer infantil. Recuperado de http://www.who.int/cancer/media/news/Childhood_cancer_day/es/

35. Ortega-Ortega, M., Montero-Granados, R., \& Jiménez-Aguilera. J. D. (2017). Differences in the economic valuation and determining factors of informal care over time: The case of blood cancer. Gaceta Sanitaria. doi: 10.1016/j.gaceta.2017.02.006

36. Patterson, J. M., Holm, K. E., \& Gurney, J. G. (2004). The impact of childhood cancer on the family: A qualitative analysis of strains, resources, and coping behaviors. Psycho-Oncology, 13(6), 390-407. doi: 10.1002/pon.761

37. Pedrón-Giner, C., Calderón, C., Martínez-Costa, C., Borraz-Gracia, S., \& Gómez-López, S. (2012). Factors predicting distress among parents/ caregivers of children with neurological disease and home enteral nutrition. Child: Care, Health and Development, 40(3), 389397. 
38. Pioli, M. F. (2010). Global and caregiving mastery as moderators in the caregiving stress process. Aging \& Mental Health, 14(5), 603612. doi: 10.1080/13607860903586193

39. Robles, R., Varela, R., Jurado, S., \& Páez, F. (2001). Versión mexicana del Inventario de Ansiedad de Beck: Propiedades psicométricas. Revista Mexicana de Psicología, 18(2), 211-218.

40. Rodríguez, G. M. (2006). Tipo de vínculo madre/hijo y desarrollo intelectual sensoriomotríz en niños de 6 a 15 meses de edad. Interdisciplinaria, 23(2), 175-201. Recuperado de http://www.redalyc.org/pdf/180/18023203.pdf

41. Romero, E. (2008). La familia del paciente paliativo. "Hablan las cuidadoras". Recuperado de http://www.humanizar.es/uploads/media/08_atencion_a_la_familia_d el_enfermo_XIII_Jorn.pdf

42. Rubira, E. A., Munhoz, M. A., Martinez, M., Barbosa, D. A., \& Silva, A. G. (2011). Cuidando del niño con cáncer: Evaluación de la sobrecarga y calidad de vida de los cuidadores. Revista LatinoAmericana de Enfermagem, 19(3), 515-522. doi: 10.1590/S01041169201100030010

43. Ruíz, A. E., \& Nava, M. G. (2012). Cuidadores: Responsabilidadesobligaciones. Enfermería Neurológica, 11(3), 163-169. Recuperado de http://www.medigraphic.com/pdfs/enfneu/ene-2012/ene123i.pdf

44. Secretaría de Salud. (2014). Comportamiento epidemiológico del cáncer en menores de 18 años. México 2008-2014. Recuperado de http://censia.salud.gob.mx/contenidos/descargas/cancer/20160601_B oletin2014_SEDP12sep16_4.pdf

45. Selye, H. (1936). A syndrome produced by diverse nocuous agents. Nature, 138, 32.

46. Sulkers, E., Tissing, W. J. E., Brinksma, A., Roodbol, P. F., Kamps, W. A., Stewart, R. E.,...Fleer, J. (2015). Providing care to a child with cancer: A longitudinal study on the course, predictors, and impact of caregiving stress during the first year after diagnosis. Psycho-Oncology, 24(3), 318-324. doi: 10.1002/pon.3652

47. van Ryn, M., Sanders, S., Kahn, K., van Houtven, C., Griffin, J. M., Martin, M.,...Rowland, J. (2011). Objective burden, resources, and other stressors among informal cancer caregivers: A hidden quality issue? Psychooncology, 20(1), 44-52. doi: 10.1002/pon.1703.

48. Zarit, S. H. (2002). Caregiver's burden. En S. Andrieu \& J. P. Aquino (Eds.), Family and professional carers: Findings lead to action (pp. 24-26). Paris: Serdi Edition y Fondation Médéric Alzheimer. 
49. Zarit, S. H., Reever, K. E., \& Bach-Peterson, J. (1980). Relatives of the impaired elderly: Correlates of feeling of burden. The Gerontologist, 20(6), 649-655. doi: 10.1093/geront/20.6.649 